\title{
Behavior modeling method for functional verification of product considering ways of usage
}

\author{
Eiji MORINAGA*, Hidefumi WAKAMATSU*, Hijiri ABIRU* and Eiji ARAI* \\ * Division of Materials and Manufacturing Science, Osaka University \\ 2-1 Yamadaoka, Suita, Osaka, 565-0871, Japan \\ E-mail: morinaga@mapse.eng.osaka-u.ac.jp
}

Received: 30 December 2015; Revised: 17 October 2016; Accepted: 6 December 2017

\begin{abstract}
Recent industrial globalization has increased importance of functional verification in product design considering various ways of usage. In particular, for shortening development time, it is desired to perform functional verification and necessary modification of the design plan in the conceptual design phase. From this point of view, a framework for such individual functional verification that can be applied to the conceptual design phase was proposed. In this framework, functions, behavior and physical phenomena caused by a way of usage are modeled by function decomposition tree, Petri net, and cause-and-effect graph, respectively. They are then integrated as an augmented Petri net, and analyses for verification are performed by qualitative simulation on the integrated model. Although fundamental feasibility of this framework was illustrated, there are issues to be discussed further for application to actual product design. A serious one is behavior modeling. In the Petri net behavior modeling, detailed ways have not been discussed and the modeling is fully dependent on the designer. The resultant model varies depending on the designers even if he/she has perceived the behavior itself the same, and verification result may be inaccurate/inadequate if the model is inappropriate. It is desirable to reduce the degree of this dependence as much as possible. In addition, it is difficult for unpracticed designers to represent behavior of the design plan as a Petri net, since, in general, designers are not familiar with Petri nets. It is necessary to make this behavior modeling easier for designers. This paper provides a detailed way of the Petri net behavior modeling so that any designers can generate a standard behavior model which works in the framework as successfully and easily as possible. Each behavior is described by a unitary Petri net and the behavior of the design plan is described as a modularly-structured Petri net composed of those units. The modular structure is beneficial to automatic generation of the Petri net behavior model from the behavior model which has been generated by the designer in advance as a flowchart, which most designers are familiar with. Detailed ways of generating a Petri net model from a flowchart model are also given. This is helpful for designers to generate the Petri net model. The proposed method was applied to an example and its effectiveness was proven.
\end{abstract}

Keywords : Behavior, Function, Conceptual design, Petri net, Flowchart

\section{Introduction}

Product design is a creative operation of generating an entity in the real world for satisfying required functions defined in the conceptual world. Relationships between the required functions and the entity are not constant but dependent on how the entity exists in the real world. Therefore, unexpected troubles, such as failing to satisfy the anticipated functions and causing undesirable events, can occur depending on the way of usage. Due to recent globalization in product development, manufacturing and business, products are utilized in various areas of the world. This resulted in increase of the number of troubles caused by using products in unexpected ways. In order to avoid such troubles, it is required to carry out the following three processes - (i) assuming various ways thoroughly, (ii) verifying whether the design plan can fulfill required functions when the product is utilized in those ways, and then (iii) modifying the design plan in advance if some functional troubles may occur. Processes (i) and (iii) depend mainly on creativity and knowledge of designers, and therefore they are hard to be automated. On the other hand, it is expected that process (ii) is automated by computers. 
Verification of a design plan is usually performed by using a prototype or design data in the embodiment design phase or the detailed design phase of the product design process. This is because it is hard to carry out the verification without concrete information about the product. However, to meet recent requirements for short-term development derived from shortening product life cycle, it is desirable to perform the verification in the earlier stage of the design process-the conceptual design phase. There have been numerous works for analyzing products or systems without concrete/detailed information about them by computers, such as qualitative reasoning (Dague, 1995; Kuipers, 1994), Modelica (Mattsson, et al., 1998; Tiller, 2001) and 1D-CAE (Sawada, 2012; Ohtomi and Hato, 2012), etc. These works focus only on behavior of the products or systems and do not consider external factors like ways of usage. Therefore, a new framework for the automated functional verification was proposed (Morinaga, et al., 2015). In this framework, functions, behavior and physical phenomena caused by a way of usage are modeled by function decomposition tree, Petri net, and cause-andeffect graph, respectively. Those models are then integrated, and it is analyzed whether some behavior is infeasible on the integrated model, which means the corresponding function cannot be fulfilled. Fundamental feasibility of this framework was proven by an example.

However, there are issues to be discussed further for practical use of the framework, and a serious one is behavior modeling. Although it has been roughly determined how to model behavior by Petri net, detailed way of the modeling has not been discussed. It is fully dependent on each designer, and the resultant model varies depending on the designers even if he/she has perceived the behavior itself the same. Therefore, it is possible that a generated model is not appropriate enough for functional verification and the verification result is inaccurate/inadequate. It is desirable to reduce the degree of this dependence as much as possible, though it would be quite difficult to get rid of the dependence. In addition, it is difficult for unpracticed designers to represent behavior of the design plan as a Petri net, since, in general, designers are not familiar with Petri nets. It is necessary to make this behavior modeling easier for designers. For these reasons, this research aims to provide an improved Petri net behavior modeling method so that any designers can generate a standard behavior model which works in the framework as successfully and easily as possible.

This paper is organized as follows. The next section outlines the framework derived from the definitions of functions and behavior and from relations between them and also between behavior and physical phenomena caused by ways of usage. Section 3 describes an improved Petri net behavior modeling method. A case study is described in Section 4, and Section 5 presents conclusions.

\section{Outline of framework for functional verification considering ways of usage}

Product design can be regarded as an operation of generating an entity which satisfies required functions. However, if the product is used in an unexpected way, some of the required functions cannot be satisfied. In other words, product design without consideration of unique ways of usage may cause unexpected functional troubles. In order to avoid such troubles, it is necessary, in addition to assuming various ways thoroughly, to systematically detect the troubles caused by the ways. This section outlines the framework for the systematic detection (Morinaga, et al., 2015) and describes its problem for practical use.

The entity of a product has its own behavior which achieves its functions (Fig. 1). As can be seen in this figure, a required function is composed of sub-functions and each of them is achieved by a set of behavior of the entity. On the other hand, a set of physical phenomena depending on ways of usage may make some of the behavior inactive. This results in failing to satisfy some sub-functions and finally the required function (Fig. 2) or generating unexpected behavior which has undesirable functional meaning. Based on this analysis, the systematic detection of troubles caused by ways of usage can be performed by the following steps - (i) modeling functions, behavior and physical phenomena caused by a way of usage, (ii) integrating those models, and (iii) making analysis on the integrated model. For the first step (i), it was proposed to model functions, behavior and physical phenomena by graphs, which are function decomposition tree (Fig. 3), Petri net (Fig. 4) and cause-and-effect graph (Fig. 5(a)), respectively. In the second step (ii), the physical phenomena model is converted into Petri net (Fig. 5(b)) and then merged with the behavior model by finding identical places. At the same time, sequences of transitions in the Petri net behavior model are related to nodes of sub-functions in the function model by a designer (Fig. 6). In the third step (iii), irregular behavior in the integrated Petri net model and its functional meanings are analyzed.

Although fundamental feasibility of this framework was shown by examples, there are issues to be discussed further for practical use of the framework. A serious one is behavior modeling. The way of modeling behavior by Petri net has been roughly determined as follows. A place represents a state of a part of the entity, and the content of the state is 


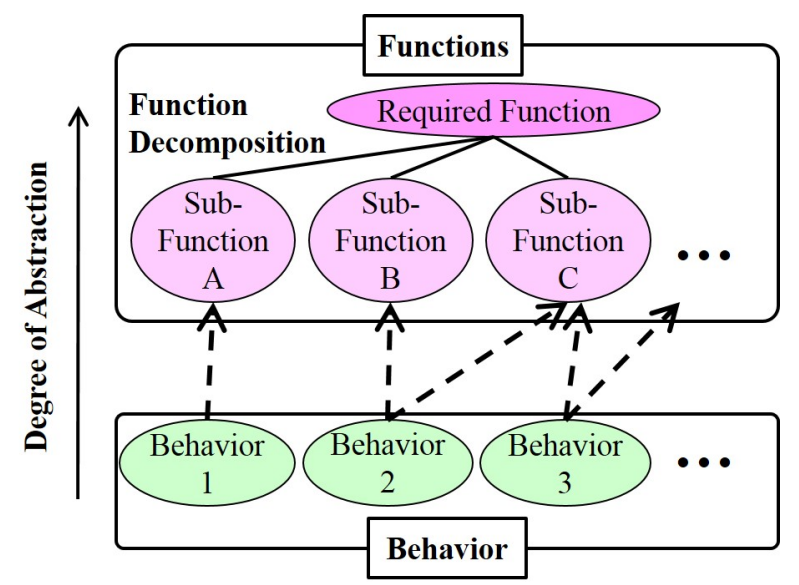

Fig. 1 Relationship between functions and behavior (Morinaga, et al., 2015).

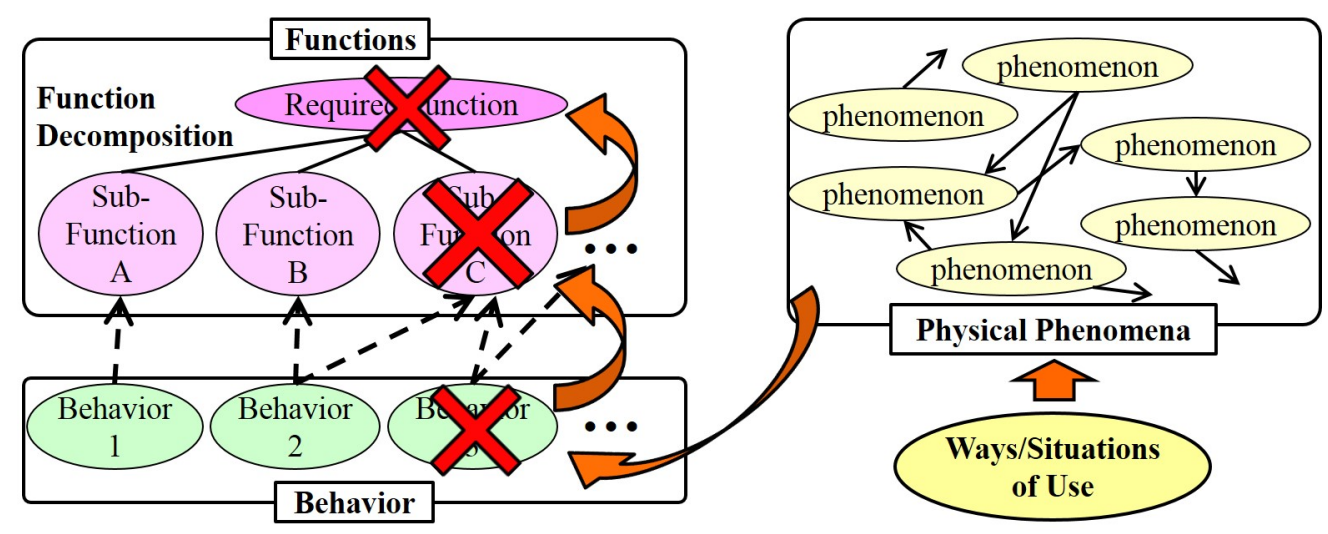

Fig. 2 Influence of ways of usage on functions via behavior (Morinaga, et al., 2015).

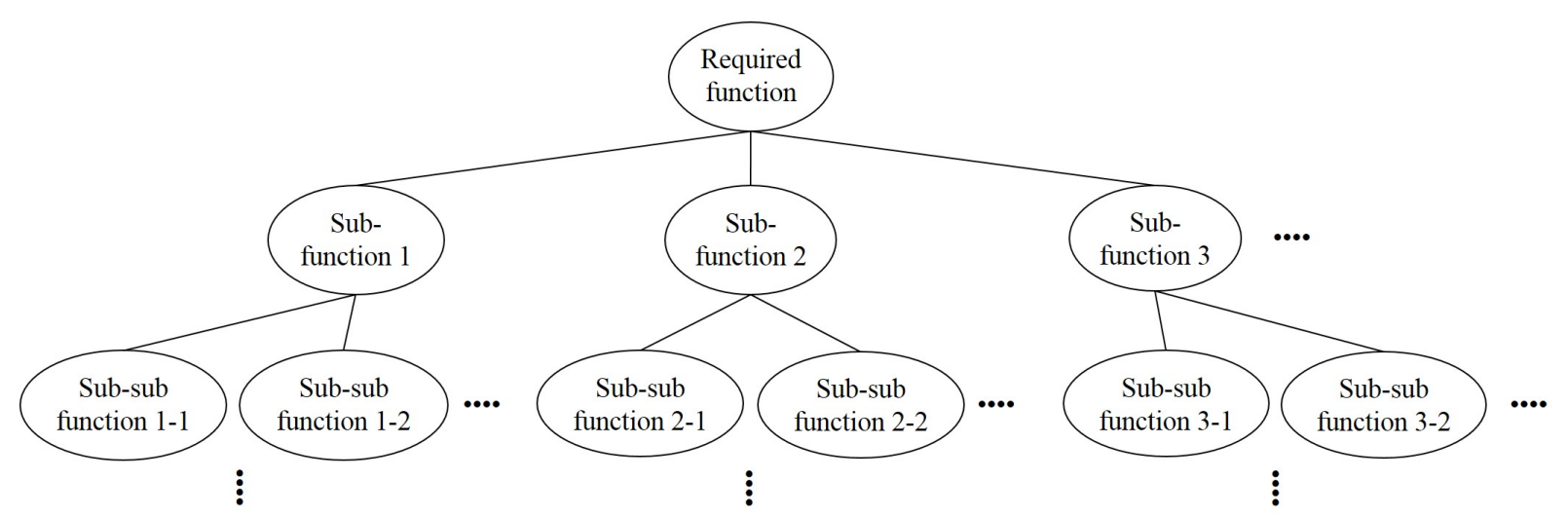

Fig. 3 Function decomposition tree model.

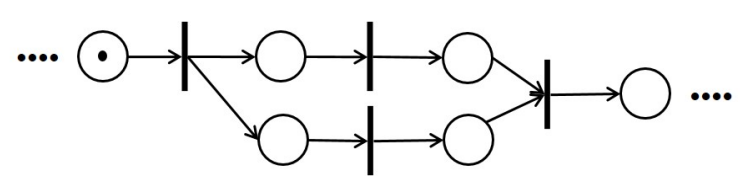

Fig. 4 Petri net behavior model. Each place stands for a possible state of a part of the entity of the product and the content of the state is described by natural language. A distribution of tokens stands for the current state of the whole product, and state change of the product represented by movement of tokens from a place to another place via a transition. 


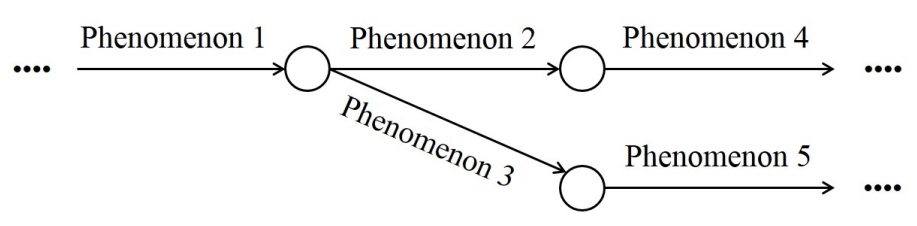

(a) Physical phenomena model by cause-and-effect graph.

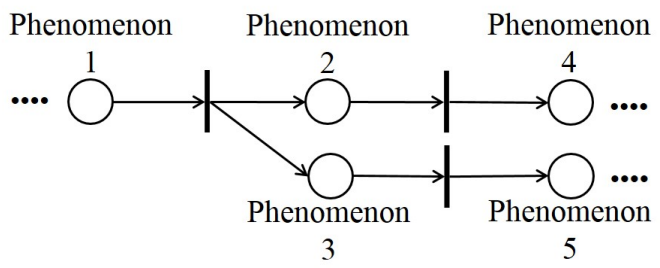

(b) Physical phenomena model by Petri net, which can be generated by converting nodes and arrows in the cause-andeffect graph into transitions and places and arrows, respectively.

Fig. 5 Physical phenomena model.

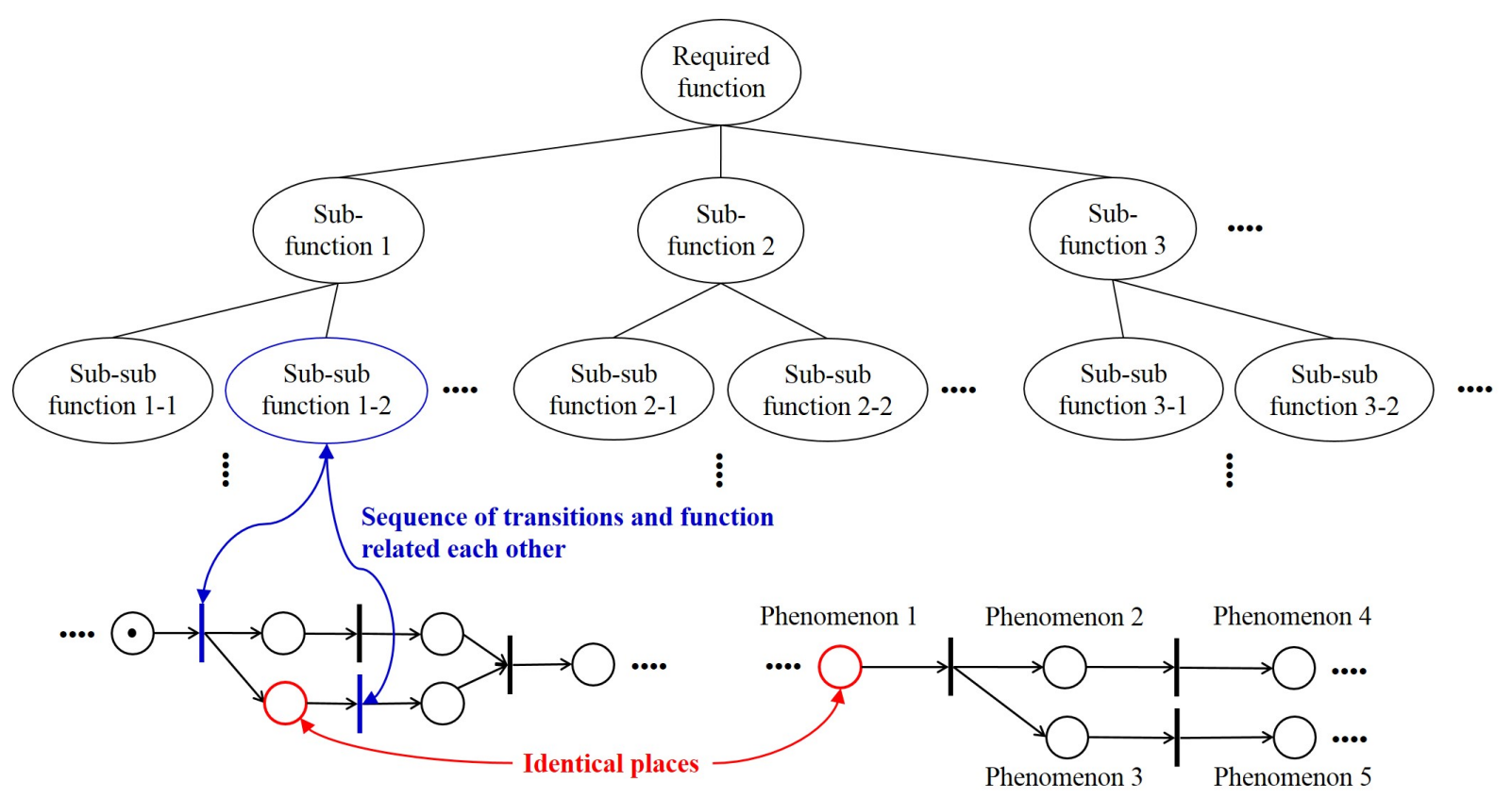

Fig. 6 Integration of the models.

described by natural language. A token in a place signifies that the state assigned to the place is "active" which means the part of the entity is in this state, and the distribution of tokens shows a set of "active" states, that is, a state of the whole entity. Behavior which was defined as a temporal change of the entity's state into its another state (Tomiyama and Yoshikawa, 1990) is represented by movement of tokens from a place to another place via a transition. However, detailed way of the modeling, e.g. how to assign a state to a place, how to connect a place to another place via a transition, etc., has not been discussed. It is fully dependent on each designer, and the resultant model varies depending on the designers even if he/she has perceived the behavior itself the same. Because results of the functional verification depend on the model, it is possible that a generated model is not appropriate enough for functional verification and the verification result may be inaccurate/inadequate. In addition, it is difficult for unpracticed designers to represent behavior of the design plan as a Petri net, since, in general, designers are not familiar with Petri net. It is necessary to improve the Petri net behavior modeling so that any designers can generate a standard model which works in the framework as successfully and easily as possible.

\section{Improved behavior modeling method}

In this section, improvement of the behavior modeling is discussed. Modularly-structured behavior modeling is derived from the characteristic of behavior that behavior occurs sequentially in general. From the point of view of supporting designers in generating a behavior model, a method of systematic conversion from a flowchart behavior model into the improved Petri net behavior model is provided. 


\subsection{Modularly-structured behavior modeling}

In general, a set of behavior does not occur at once but each behavior occurs sequentially. It is therefore natural to regard each behavior as a unit and generate the whole behavior model by connecting those units. As described in Section 2 , based on the definition of behavior, each behavior can be described by movement of a token from a place to another place via a transition. In order to generate the whole behavior model by connecting these units, it is necessary to add some elements of a Petri net as an "interface" to this unit. Figure 7(a) shows a unitary Petri net considering these points. The black elements represents the behavior and the red elements are for the interface. When the transition " $t 1$ " fires, the token in place "p3" moves to "p4" which signifies the behavior. At the same time, the token in "p1" moves to "p2" which means the behavior has completed and the next behavior can occur (Fig. 7(b)). In the case that there are a set of behavior that occur at once, it is possible to represent them by this unitary Petri net by adding places concerning those behavior (Fig. 7(c)). By connecting these unitary Petri nets, it is possible to generate the whole behavior model systematically as shown in Fig. 8. By generating the model in this way, the structure of the whole model is standardized and does not vary depending on designers.

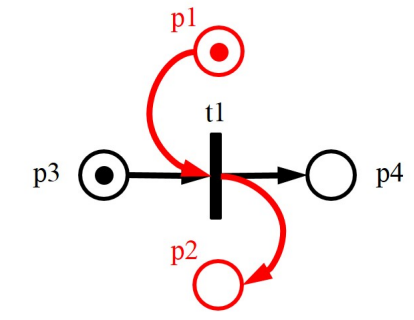

(a) The state before firing of the transition.

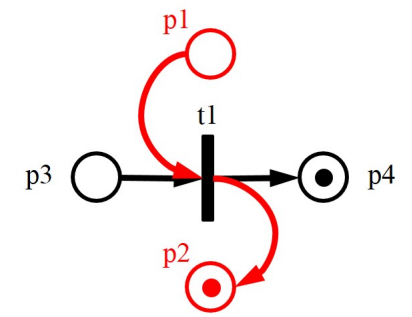

(b) The state after firing of the transition.

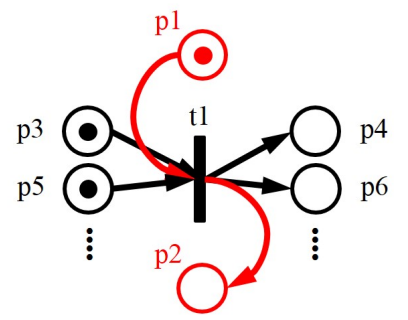

(c) Description of a set of behavior that occur at the same time.

Fig. 7 Unitary Petri net.

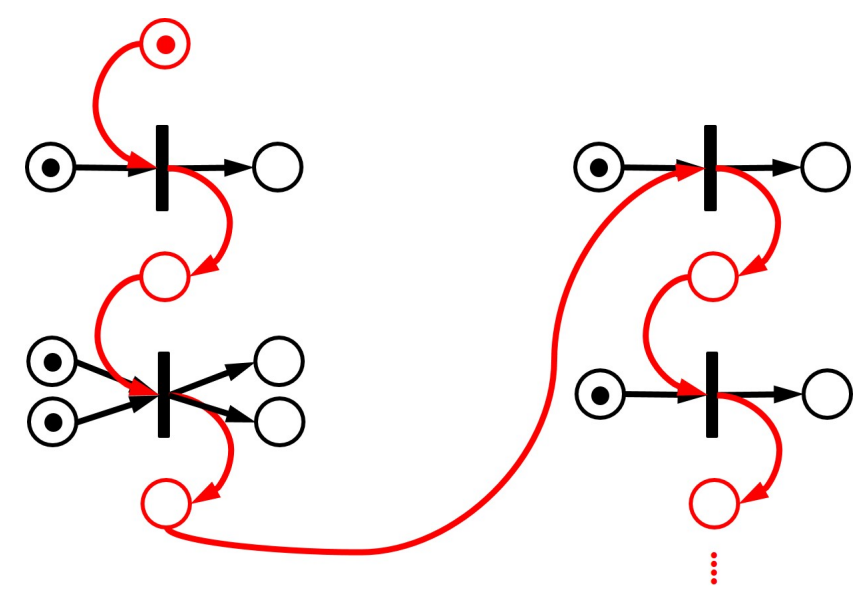

Fig. 8 Behavior model generation by connecting the unitary Petri nets.

\subsection{Support of behavior modeling by flowchart}

The modularly-structured behavior modeling method allows designers to generate a behavior model systematically, and then their load in modeling is reduced. However, many designers are not familiar with Petri net, and the modeling using Petri net itself is a burden for them. It is desirable to enable designers to generate a behavior model without dealing with Petri net directly. A natural approach is to represent a unitary behavior by a modeling method other than Petri net and then convert it systematically into a Petri net. Considering that it is in widespread use in various field and most designers are familiar with it, flowchart is regarded as a promising candidate for the intermediate modeling method.

A flowchart is composed of flow lines and nodes, and has a structure in which units consisted with two flow lines and a node are connected each other. This characteristic is shared with the modularly-structured Petri net modeling. Therefore, if the nodes can be transformed into Petri net equivalently, it is possible to convert each unit into the unitary Petri net (Fig. 9) and generate the Petri net behavior model by the modularly-structured modeling method. The transformation of a node 
into Petri net can be performed as follows. In a process, predefined process or input/output, state changes take place. That is, they are behavior themselves and a node of them can be transformed as shown in Fig. 10. A decision, which controls the flow depending on a condition, activates the path whose condition is satisfied. This role can be represented in Petri net by introducing a place signifying the condition which has both incoming and outcoming arcs connected to a transition, and a node of decision can be transformed as shown in Fig. 11. In this transformation, it is necessary to assign a token to the condition which is satisfied by default. Terminals and page connectors are transformed as a place as shown in Fig. 12.

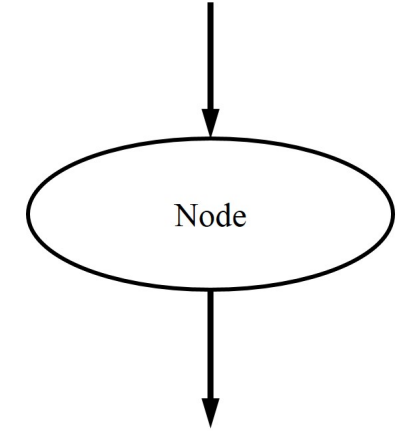

Unit in a flowchart

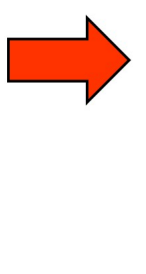

Unitary Petri net in the modularlystructured modeling method

Fig. 9 Conversion of a unit in a flowchart into a unitary Petri net.
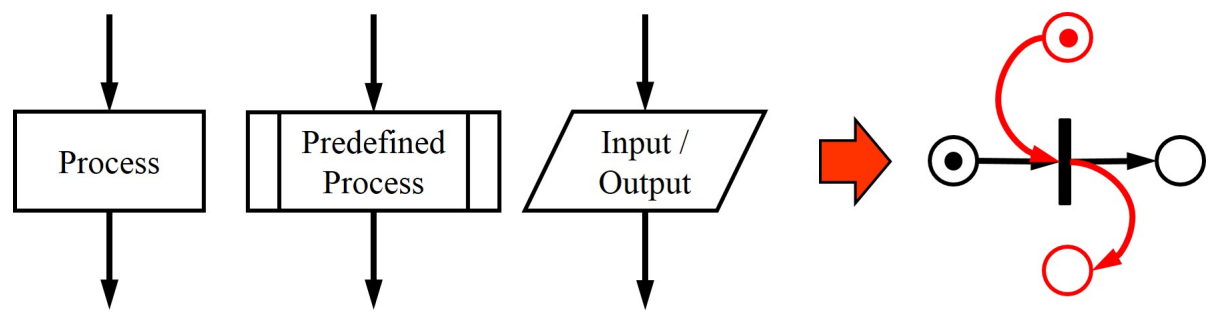

Fig. 10 Conversion of a unit with a node of process, predefined process and input/output into a unitary Petri net.

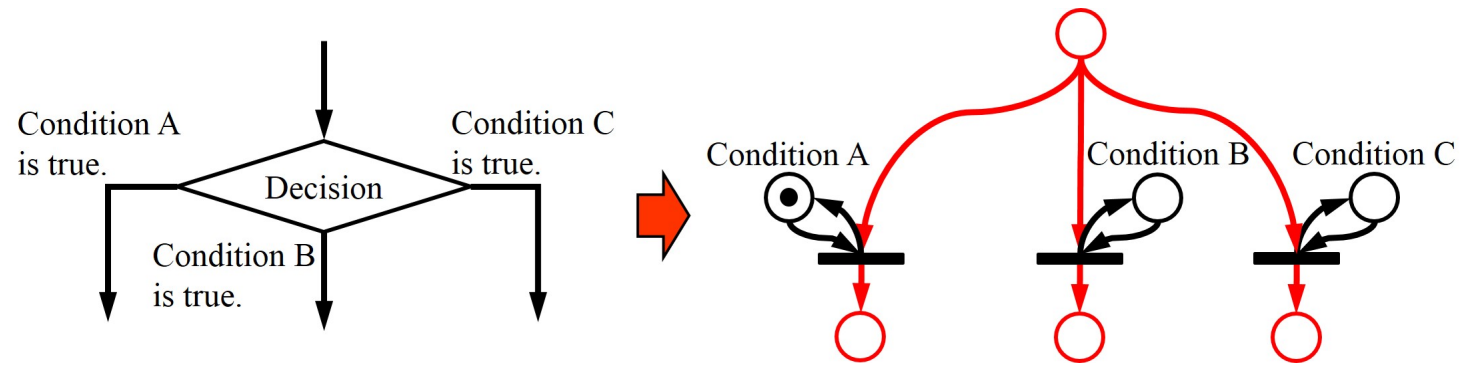

Fig. 11 Conversion of a unit with a node of decision into a unitary Petri net. Black places represent the conditions in the decision. A token needs to be assigned to the condition satisfied by default ("Condition A" in this figure).

Once each unit in a flowchart is transformed into a unitary Petri net, the units are connected each other as specified in the flowchart. This is basically performed by merging the places for interface as shown in Fig. 8. This is however incomplete and generate an invalid Petri net if there is a loop in the flowchart. If there is a loop (Fig. 13(a)), the Petri net shown in Fig. 13(b) is generated. In this Petri net, the tokens in "p1" and "p3" move to "p2" and "p4" by firing of " $t 1$ ", and then the token in "p2" moves to "p1" by firing of " $t 3$ ". However, "t1" cannot fire this time and the loop does not work, since "p3" does not have a token. This problem can be solved by inserting a unit for "resetting" as shown in Fig. 13(c). In this modified Petri net, the movement of a token from "p3" to "p4" is reset at the same time when the token in "p8" moves to "p1", and therefore "t1" can fire again and the loop works. Note that this inserting can be automatically performed by detecting a loop in the flowchart and therefore the valid Petri net can be generated systematically.

In order to convert the behavior model described by a flowchart into a Petri net, it is necessary not only to transform 


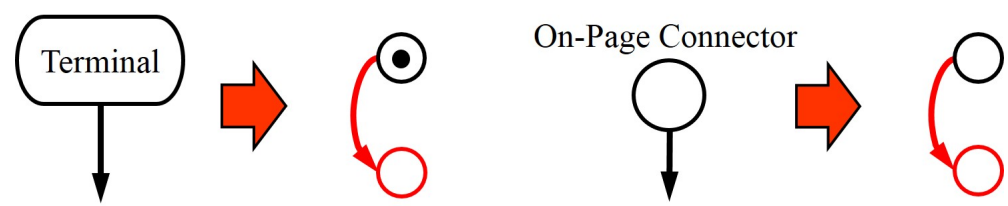

(a) Conversion of a unit with a node of start terminal and on-page connector.

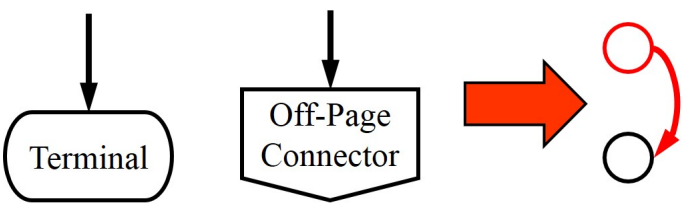

(b) Conversion of a unit with a node of end terminal and off-page connector.

Fig. 12 Conversion of a unit with a node of terminal and connector into a unitary Petri net.

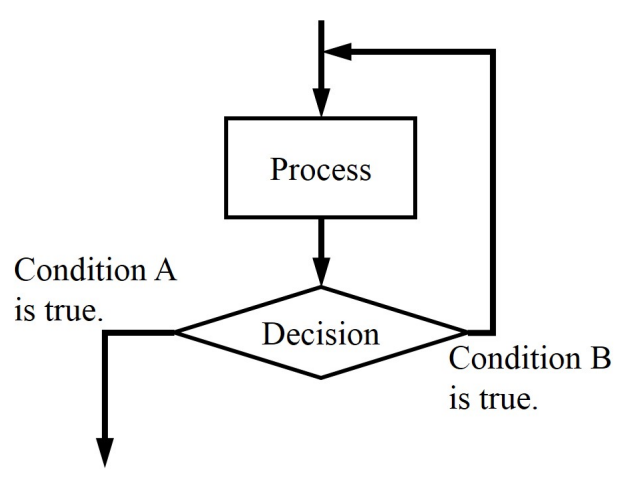

(a) Flowchart with a loop.

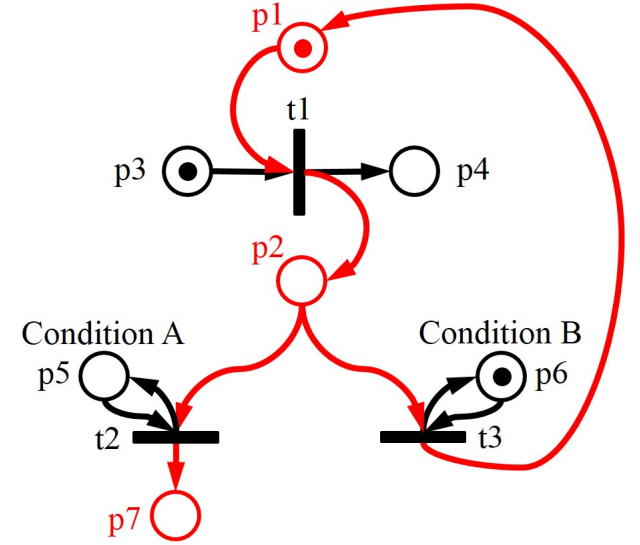

(b) Petri net generated by merging the places for interface.

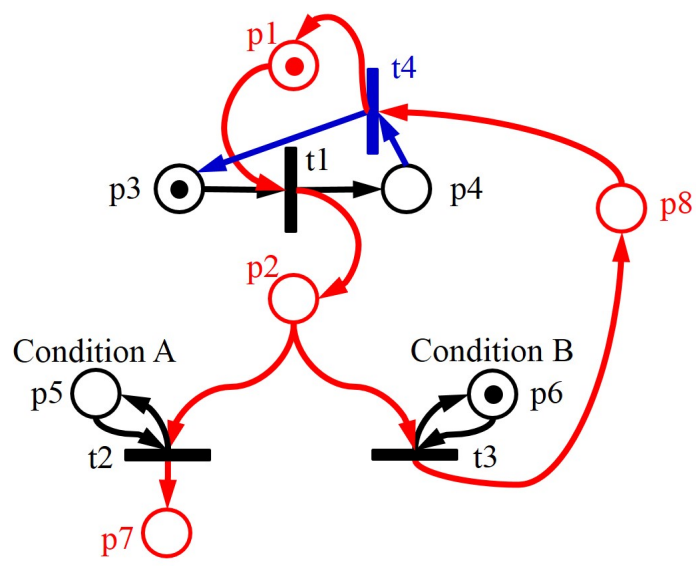

(c) Modified Petri net by inserting a unit for resetting.

Fig. 13 Conversion of a flowchart with a loop into a Petri net. 
its structure but also to transfer the information included in it. From this point of view, it is required to devise ways of describing the information in the flowchart. A simple way is to make the designer describe it by natural language with clarifying each state and condition. For example, if a process is described in the format " $\{$ object; the previous state (of the object); the subsequent state (of the object)\}", it is possible to automatically transfer the information to the places of state in the Petri net (Fig. 14(a)). If a decision is described in the format "\{object; state 1; state 2; state 3\}", it is also possible to automatically transfer the information to the places of condition in the Petri net (Fig. 14(b)). If the decision is the yes-no decision and described in the format " $\{$ object; state $\}$ ", the information can be transferred systematically to the Petri net (Fig. 14(c)).

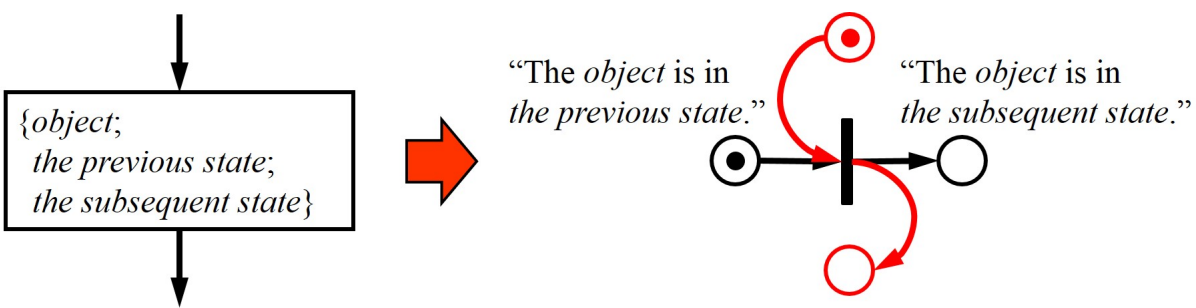

(a) Automatic transfer of the information in a node of process.
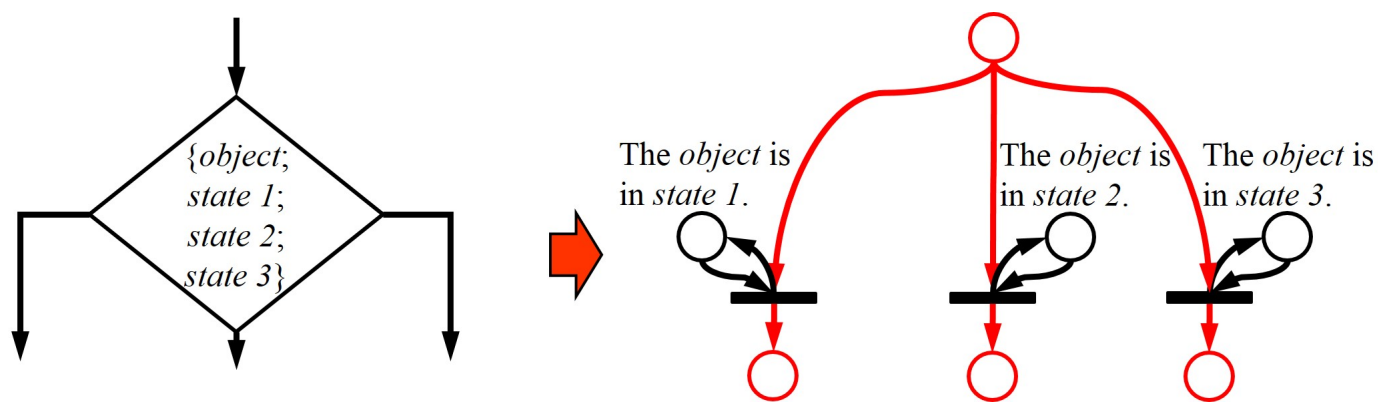

(b) Automatic transfer of the information in a node of decision.

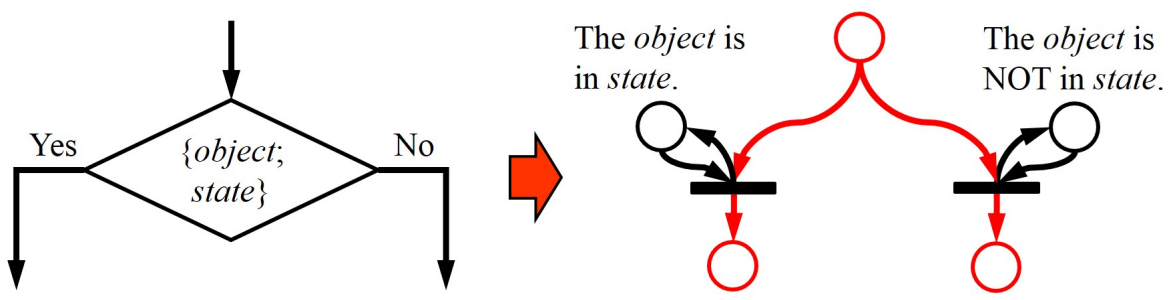

(c) Automatic transfer of the information in a node of yes-no decision.

Fig. 14 Automatic transfer of the information in a node.

\section{Case study}

In order to verify that it is possible to generate a Petri net behavior model easily and automatically by the proposed method and also that the model surely works in the framework, the proposed method was applied to a case of functional troubles in a computerized aircraft caused by wrong way of usage, which resulted in a terrible accident (Aircraft Accident Investigation Commission of Japan, 1996). The abstract of this case is as follows. In the landing operation, the copilot activated go-around mode of the automatic control system (ACS) by mistake. The captain in command and copilot tried to inactivate the mode but they failed to do that. However, they erroneously believed that the mode was inactivated, and kept the landing operation. As a result, the ACS inclined the trimmable horizontal stabilizer (THS) to lift the nose of the aircraft, and, at the same time, the pilots inclined the elevator to lower the nose of it (Fig. 15). Staying in this state for a few minutes made the THS and elevator extremely inclined. The pilots then decided to perform go-around and stopped inclining the elevator. This allowed the extremely-inclined THS to lift the nose enormously, which resulted in stall of the aircraft.

This case can be regarded as an example where the function of the ACS for go-around was not satisfied due to the wrong utilization of it by the pilots. Therefore, the behavior of the ACS was modeled by the proposed method. 


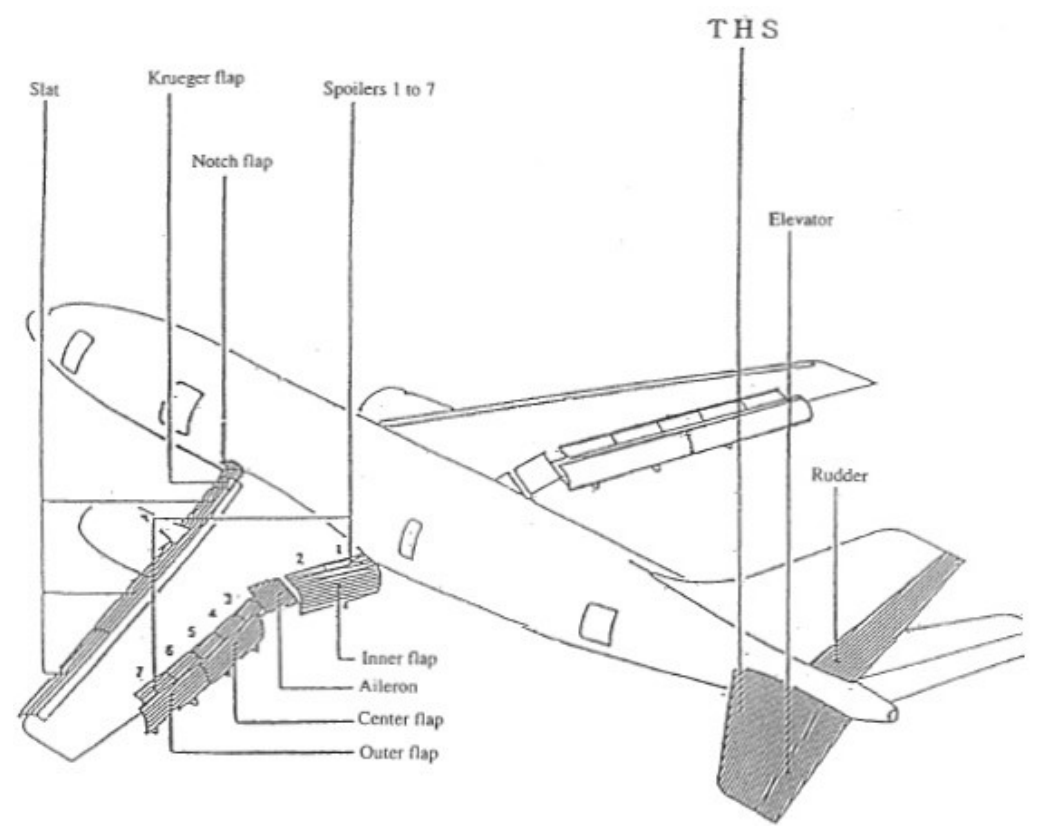

Fig. 15 THS and elevator of the aircraft (Aircraft Accident Investigation Commission of Japan, 1996, p.A15).

(Although the ACS is only a part module of the aircraft which is a large-scaled complex system, behavior of only the ACS was modeled in this case study because it was a key of this accident and it was sufficient to consider only the ACS for detecting the trouble as described below. If it is necessary to consider whole of a complex system to detect a trouble on it, it is, of course, required to model behavior of the whole system.) The behavior was simplified and its model was given as a flowchart as shown in Fig. 16 and Table 1. (It is possible that there are insufficient information and many uncertain attributes, especially in the early stage of product design. However, it is still possible to model behavior of an entity in the level of detail that can be achieved with the given information and attributes and therefore to detect a trouble which can be analyzed in that level. Considering this point and readability, the behavior was simplified as much as possible.) This model was converted to the Petri net model shown in Fig. 17 and Table 2 automatically and instantly by the proposed method, which was implemented on a workstation by coding in the $\mathrm{C}$ language. This Petri net model is generated by any designer as long as he/she generates the same flowchart model. For the reason of consistency, the physical phenomena caused by the wrong utilization was not modeled by the cause-and-effect graph modeling method but by the modularly-structured Petri net modeling method as shown in Fig. 18 and Table 3. These two models were integrated by merging "p9" and "p21" which have same information, that is, stand for the same state of the entity. (This framework presupposes that places having the same information represent the same state of the entity. Two different states which should be distinguished in detailed level where multiple attributes are handled can be regarded as the same state in rough level. If the designer needs to distinguish those states to obtain meaningful results, he/she must describe the behavior with a sufficiently-high degree of detail, that is, with sufficient number of places for representing those attributes.) This integration generates the Petri net shown in Fig. 19, and simulation on it detected that the transition between "p9 (p21)" and "p10" fails to fire when the transition between "p21 (p9)" and "p22" fires earlier, which means that the function of the ACS for lifting the nose of the aircraft is not satisfied. In addition, the simulation stops because all transitions are deadlocked, which enables alerting the designer to the trouble automatically.

Without the proposed method, it is possible that another behavior model is generated by the designer, since there was only the rough guideline on the Petri net behavior modeling as described in Section 2. Figure 20 shows an example of such behavior models for this case. This model represents the behavior of the ACS as well in which increasing the thrust force of the engine, inclination of THS and the pitch angle of the body are performed sequentially and repeatedly, though the number of tokens in places "p5", "p7", "p9" increases on each repetition. If the phenomena model shown in Fig. 18 is generated and merged with this behavior model, the integrated model shown in Fig. 21 is obtained. In this integrated Petri net, the transition between "p9 (p21)" and "p10" can fire even after firing of the transition between "p9 (p21)" and "p22" because a token can re-appear in "p9" by firing the transitions among "p5"-"p9". Therefore, the simulation can continue and therefore the trouble cannot be detected automatically without additional processes such as finding the infinite loop or finding the difference between the simulation result and the default one obtained in the behavior model in Fig. 20. 


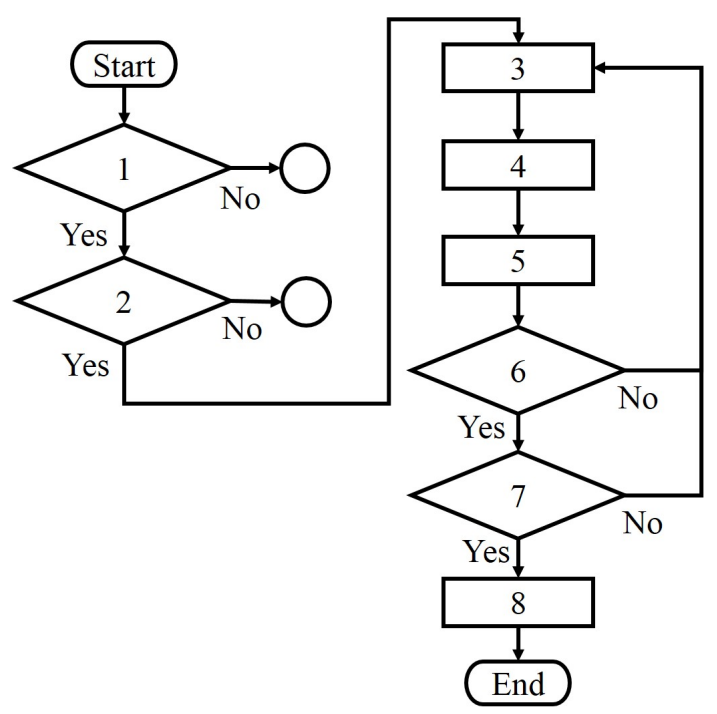

Fig. 16 Behavior model of the ACS given as a flowchart. The numbers stand for the ID number of each element.

Table 1 Information included in each node in Fig. 16.

\begin{tabular}{l|l|l|l}
\hline ID No. & Information & ID No. & Information \\
\hline 1 & \{inclination of the slats and flaps; $15[\mathrm{deg}]\}$ & 2 & \{the "go-around mode"; activated\}. \\
\hline 3 & $\{$ the thrust force of the engine; $x[\mathrm{~N}] ; x+\Delta x[\mathrm{~N}]\}$ & 4 & \{inclination of THS; $a[\mathrm{deg}] ; a+\Delta a[\mathrm{deg}]\}$ \\
\hline 5 & the pitch angle of the body; $p[\mathrm{deg}] ; p+\Delta p[\mathrm{deg}]\}$ & 6 & $\{$ the pitch angle of the body; $\alpha$ [deg] \\
\hline 7 & $\{$ the altitude; $h[\mathrm{~m}]\}$ & 8 & $\{$ the "go-around" process; incomplete; complete \\
\hline
\end{tabular}

Furthermore, it is impossible to represent some states of the ACS by the behavior model. For example, in actual behavior of the ACS, the state where both the thrust force of the engine and the inclination of THS is increased. However, it is impossible to represent this state by the behavior model. As this example shows, this behavior model is unnatural from the point of view of accuracy.

The simulation result by the proposed method was effective for detecting that a trouble may occur. However, it was not strictly accurate, since it does not indicate that the pitch angle of the body does not change as occurred actually. If it is possible to find that the transitions between "p9" and "p10" and between "p21" and "p22" have the opposite meaning each other, this problem can be solved by connecting the preceding places of these transitions by adding a new transition and a new place as shown in Fig. 22. In this model, the new transition can fire before firing of those two transitions and the simulation stops. This indicates a trouble. Although the process of finding such transitions depends on the designer at the current stage, it is possible to perform the accurate verification in essence.

\section{Conclusion}

In the framework for the automated functional verification considering ways of usage, the behavior model, which is represented as a Petri net, has a decisive influence on the quality of the verification result. This paper has provided a detailed way of the Petri net behavior modeling so that any designers can generate a standard behavior model which works in the framework as successfully and easily as possible. Each behavior was described by a unitary Petri net and the whole behavior of the design plan was described as a modularly-structured Petri net composed of those units. The modular structure is beneficial to automatic generation of the Petri net behavior model from the behavior model which has been generated the designer in advance as a flowchart, which most designers are familiar with. Detailed ways of generating a Petri net model from a flowchart model are also given. This is helpful for designers to generate the Petri net model. The proposed method was applied to an example and its effectiveness was proven.

The Petri net behavior model obtained by the proposed method does not vary depending on designers as long as they can generate the same flowchart model, namely, as long as they perceive the behavior the same. However, the method does not help designers to perceive the behavior appropriately. In this sense, the proposed method does not entirely solve the problem that the behavior modeling depends on designers. Further augmentation is desired and discussions from the point of view of national language processing, ontology, etc. may be helpful.

The approach to obtain Petri nets by transformation of another models has been actively discussed in the research 


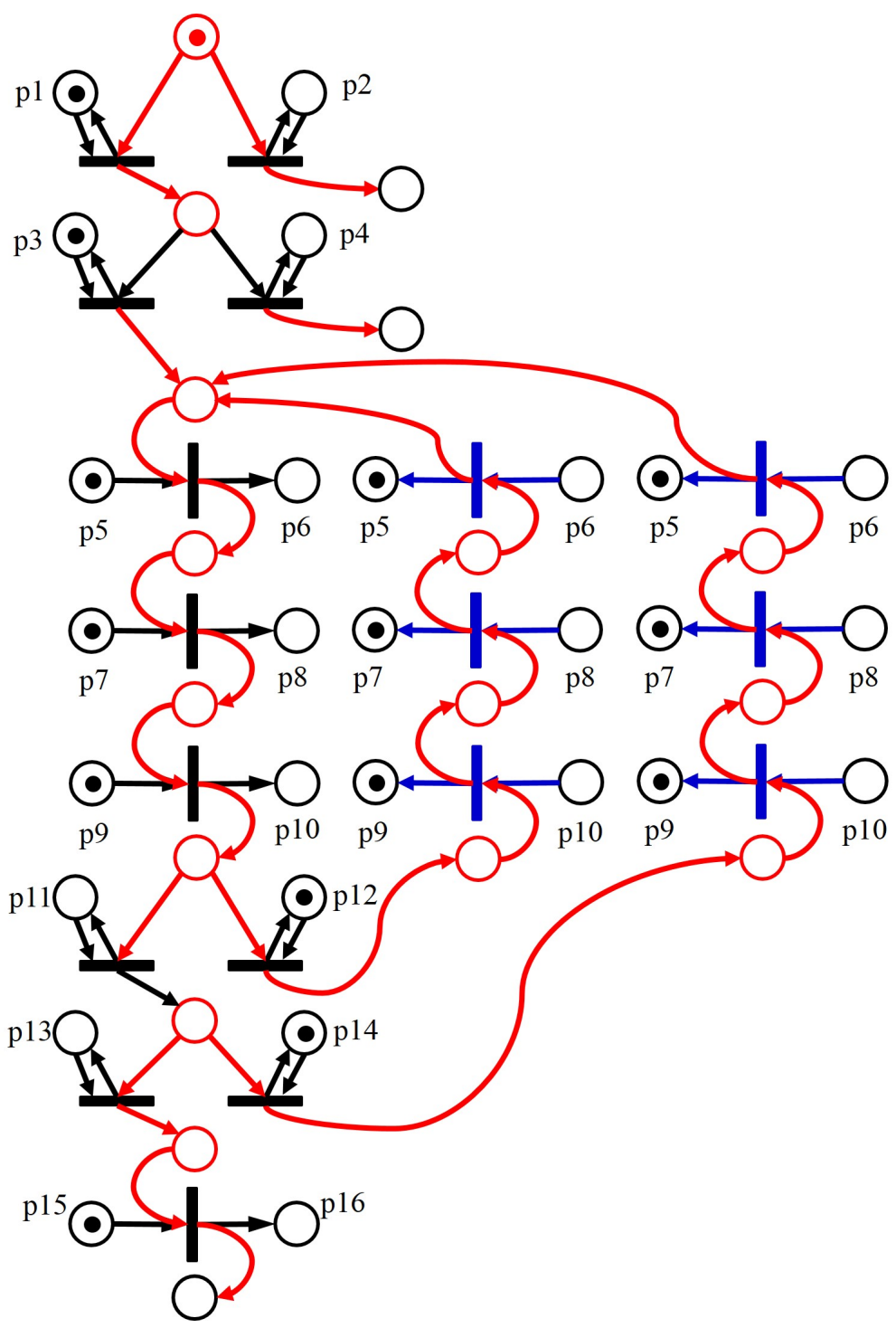

Fig. 17 Behavior model of the ACS obtained by the proposed method. (To make this model easier to understand, places "p5"-"p10" are duplicated. If a token in "p5" moves to "p6" by firing a transition, the tokens in the other two "p5" also move to the other two "p6" regardless of the transitions between them.)

Table 2 Information transferred to each place in Fig. 17.

\begin{tabular}{l|l|l|l}
\hline No. & Information & No. & Information \\
\hline p1 & Inclination of the slats and flaps is 15 [deg]. & p2 & Inclination of the slats and flaps is not 15 [deg]. \\
\hline p3 & The "go-around mode" is activated. & p4 & The "go-around mode" is not activated. \\
\hline p5 & The thrust force of the engine is $x[\mathrm{~N}]$. & $\mathrm{p} 6$ & The thrust force of the engine is $x+\Delta x[\mathrm{~N}]$. \\
\hline p7 & Inclination of THS is $a[\mathrm{deg}]$. & $\mathrm{p} 8$ & Inclination of THS is $a+\Delta a[\mathrm{deg}]$. \\
\hline p9 & The pitch angle of the body is $p[\mathrm{deg}]$. & $\mathrm{p} 10$ & The pitch angle of the body is $p+\Delta p[\mathrm{deg}]$. \\
\hline p11 & The pitch angle of the body is $\alpha[\mathrm{deg}]$. & $\mathrm{p} 12$ & The pitch angle of the body is not $\alpha$ [deg]. \\
\hline p13 & The altitude is $h[\mathrm{~m}]$. & $\mathrm{p} 14$ & The altitude is not $h[\mathrm{~m}]$. \\
\hline p15 & The "go-around" process is incomplete. & $\mathrm{p} 16$ & The "go-around" process is complete. \\
\hline
\end{tabular}




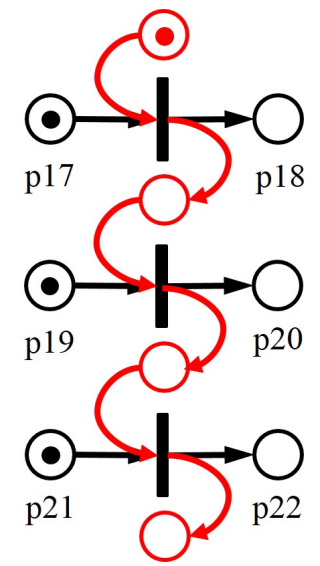

Fig. 18 Petri net model of phenomena caused by the wrong utilization.

Table 3 Definition of places in Fig. 8.

\begin{tabular}{l|l|l|l}
\hline No. & State & No. & State \\
\hline p17 & Inclination of the control stick is $\tau$ [deg]. & p18 & Inclination of the control stick has inclination of $\tau-\Delta \tau[\mathrm{deg}]$. \\
\hline p19 & Inclination of the elevator is $\varepsilon$ [deg]. & p20 & Inclination of the elevator is $\varepsilon-\Delta \varepsilon$ [deg]. \\
\hline p21 & The pitch angle of the body is $p$ [deg]. & p22 & The pitch angle of the body is $p-\Delta p$ [deg]. \\
\hline
\end{tabular}

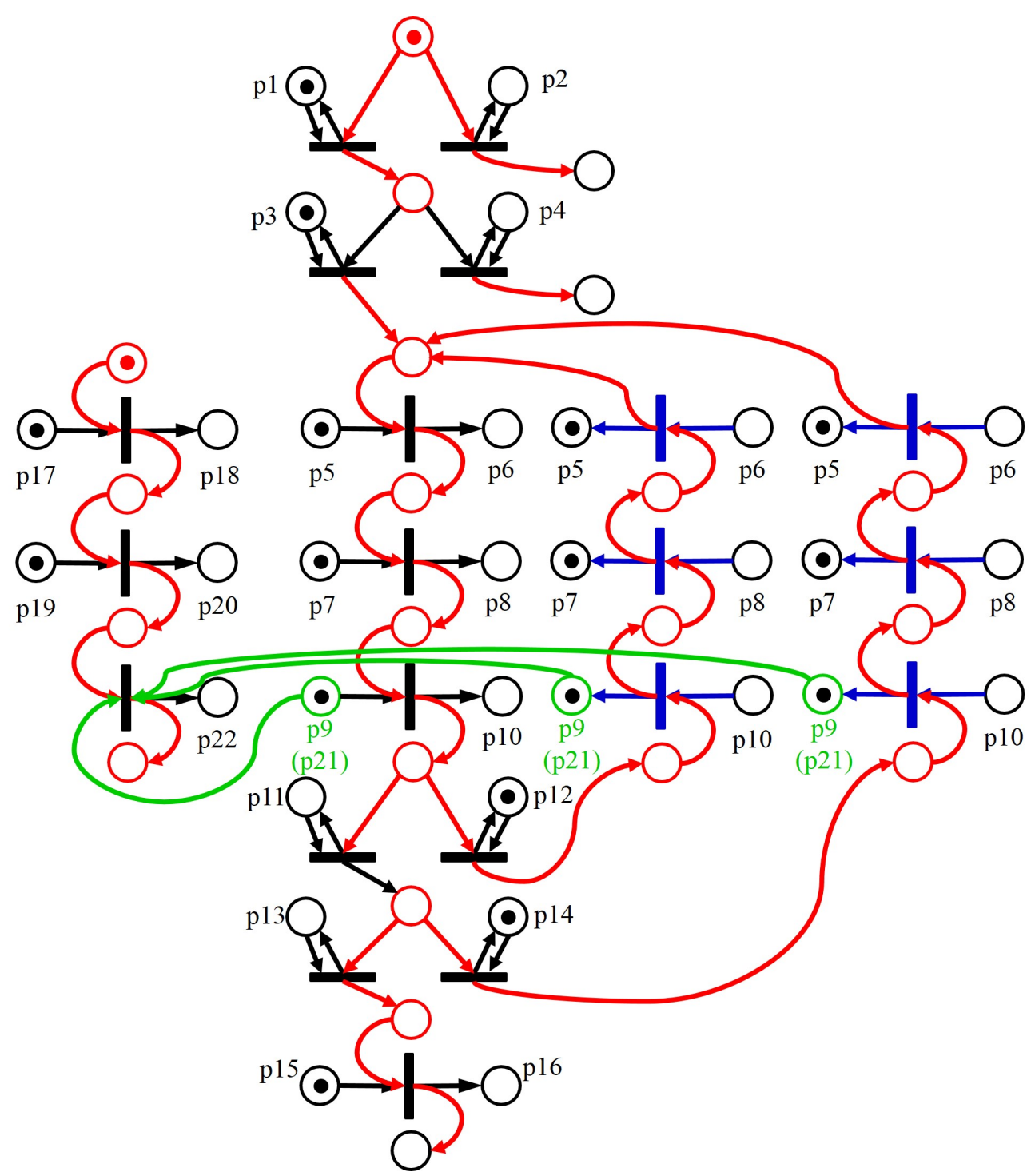

Fig. 19 Integrated model of the behavior and the phenomena models by merging the places having the same information. 


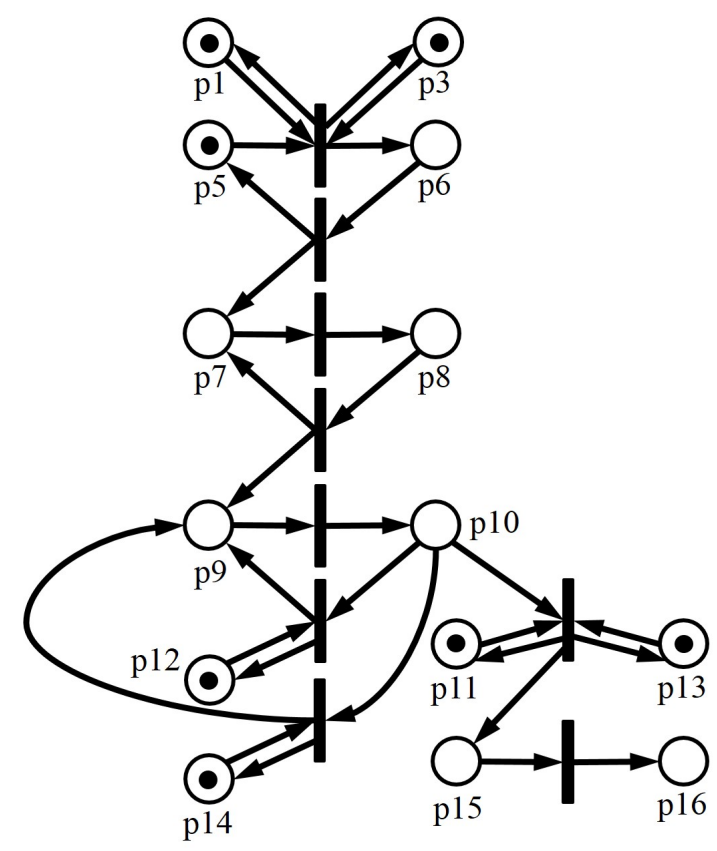

Fig. 20 An example of behavior model generated without the proposed method.

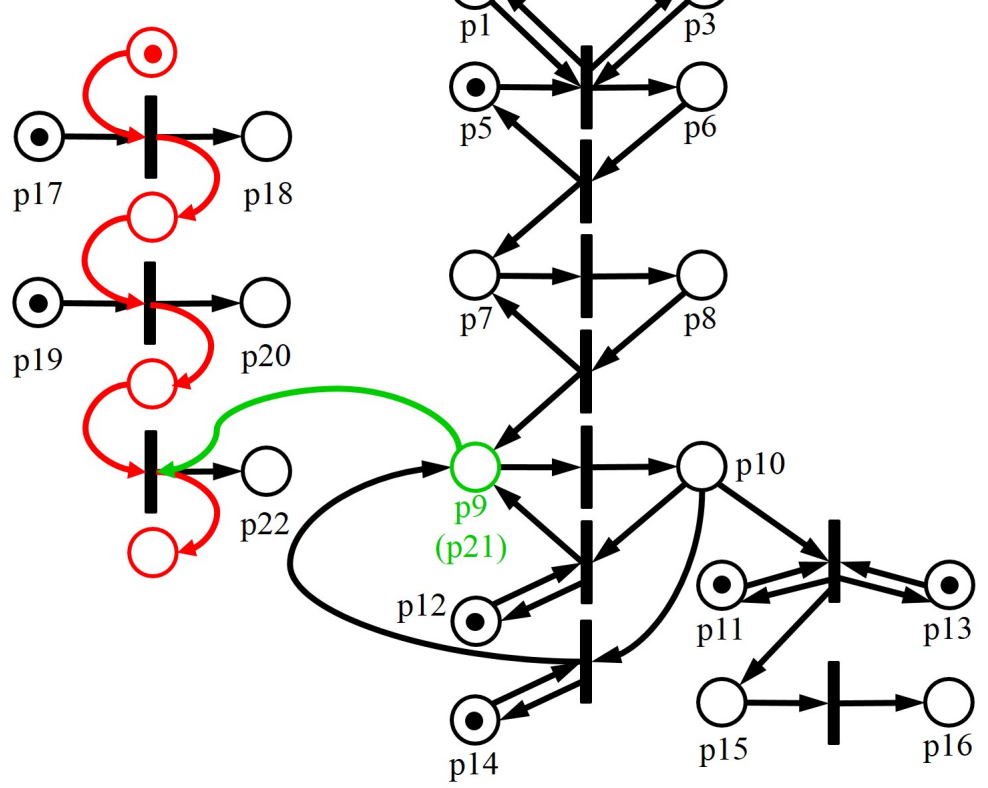

Fig. 21 Integrated model of the behavior model in Fig. 20 and the phenomena model by merging the places having the same information. 


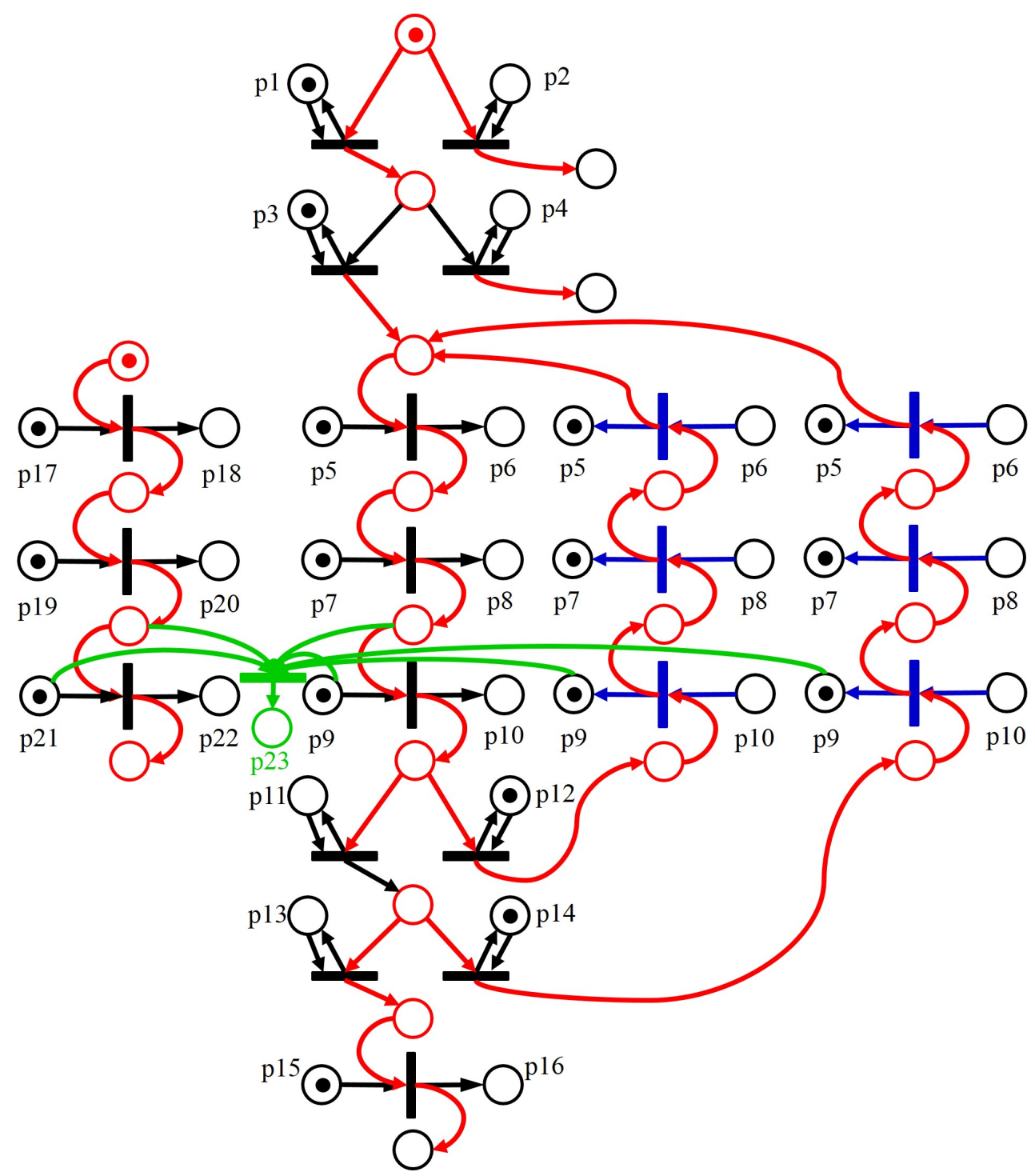

Fig. 22 Integration of the behavior and the phenomena models by focusing on the transitions having the opposite meaning each other. If the new transition (green one) fires, the tokens in "p9" and "p21" move to the new place "p23", and then no transitions can fire anymore. 
field of software engineering for exploiting UML (Unified Modeling Language) and SysML (System Modeling Language) (Agarwal, 2013; Alhroob, et al., 2014; Bowles and Meedeniya, 2010; Carneiro, et al., 2008; Emadi and Shams, 2009; Fernandes, et al., 2007; Hu and Shatz, 2004; Kessentini, et al., 2010; López-Grao, et al., 2004; Pais, et al., 2014; Rahim, et al., 2014; Rahim, et al., 2013; Ribeiro and Fernandes, 2006; Saputra, et al., 2010; Yang, et al., 2011). Those researchers proposed methods for transforming diagrams of UML and SysML such as activity diagrams and sequence diagrams in order to take advantage of theoretical results and features of Petri nets. The concept of the method proposed in this paper is basically common to those conventional methods. But, unlike those methods, the proposed method makes it possible to generate Petri nets with clear description of each state in a change of states in a process, which is critical to adequate verification in the functional verification framework. On the other hand, UML and SysML have much more advantage in representing software/hardware systems accurately than flowchart. Enhancement for taking this advantage of UML and SysML is desirable for realizing verification of design plans from various points of view.

As described in section 4, it is necessary to develop a method for finding transitions having the opposite meaning each other systematically. The method would be helpful for generating a function model and integrating it with the behavior model systematically. In addition, automatic generation of the physical phenomena model is also necessary, since it must be generated for each way of usage. These four issues will be discussed in future works.

\section{Acknowledgement}

We thank the members of Production Technology Laboratory for Device of Creation/Storage/Conservation Energy, Osaka University for their advice and cooperation, and Panasonic Corporation for its support.

\section{References}

Agarwal, B., Transformation of UML Activity Diagrams into Petri Nets for Verification Purposes, International Journal of Engineering and Computer Science, Vol.2 (2013), pp.798-805.

Aircraft Accident Investigation Commission of Japan, Japan Ministry of Transport, Aircraft accident investigation report, available from [http://www.mlit.go.jp/jtsb/eng-air_report/B1816.pdf], (1996).

Alhroob, A. and Yousef, N., Transforming UML State Machine Diagram to High Level Petri Net Using Genetic Algorithm, Lecture Notes on Software Engineering, Vol.2 (2014), pp.243-246.

Bowles, J. and Meedeniya, D., Formal Transformation from Sequence Diagrams to Coloured Petri Nets, Proceedings of 2010 Asia Pacific Software Engineering Conference (2010), pp.216-215.

Carneiro, E., Maciel, P. and Callow, G., Mapping SysML State Machine Diagram to Time Petri Net for Analysis and Verification of Embedded Real-Time Systems with Energy Constraints, Proceedings of 2008 International Conference on Advances in Electronics and Micro-electronics (2008), pp.1-6.

Dague, P., Qualitative reasoning: A survey of techniques applications, AI Communications, Vol.8, Nos.3-4 (1995), pp.119-192.

Emadi, S. and Shams, F., Transformation of usecase and sequence diagrams to petri nets, Proceedings of 2009 ISECS International Colloquium on Computing, Communication, Control, and Management (2009), pp.399-403.

Fernandes, J. M., Tjell, S., Jørgensen, J. B. and Ribeiro, Ó, Designing Tool Support for Translating Use Cases and UML 2.0 Sequence Diagrams into a Coloured Petri Net, Proceedings of the 6th International Workshop on Scenarios and State Machines (2007), Paper No.2.

Hu, Z. and Shatz, S. M., Mapping UML Diagrams to a Petri net Notation for System Simulation, Proceedings of the 16th International Conference on Software Engineering and Knowledge Engineering (2004), pp.213-219.

Kessentini, M., Bouchoucha, A., Sahraoui, H. and Boukadoum, M., Example-Based Sequence Diagrams to Colored Petri Nets Transformation Using Heuristic Search, Modelling Foundations and Applications (2010), pp.156-172, Springer-Verlag Berlin Heidelberg.

Kuipers, B., Qualitative Reasoning: Modeling and Simulation with Incomplete Knowledge (1994), The MIT Press.

López-Grao, J. P., Merseguer, J. and Campos, J., From UML Activity Diagrams to Stochastic Petri Nets: Application to Software Performance Engineering, Proceedings of the 4th International Workshop on Software and Performance (2004), pp.25-36.

Mattsson, S. E., Elmqvist, H. and Otter, M., Physical system modeling with Modelica, Control Engineering Practice, Vol.6 (1998), pp.501-510. 
Miles, L. D., Techniques of Value Analysis and Engineering (1972), McGraw-Hill.

Morinaga, E., Akasaka, T., Wakamatsu, H., Arai, E. and Abiru, H., Framework for Functional Verification in Product Design Considering Ways and Situations of Use, Transactions of the Institute of Systems, Control and Information Engineers, Vol.28, No.3 (2015), pp.116-122.

Ohtomi, K. and Hato, T., Design innovation applying 1DCAE, TOSHIBA REVIEW, Vol.67, No.7 (2012), pp.7-10. (In Japanese)

Pais, R., Barros, J. and Gomes, L., From SysML State Machines to Petri Nets Using ATL Transformations, Proceedings the 5th Doctoral Conference on Computing, Electrical and Industrial Systems (2014), pp.227-236.

Rahim, M., Boukala-Ioualalen, M. and Hammad, A., Petri Nets Based Approach for Modular Verification of SysML Requirements on Activity Diagrams, Proceedings of the International Workshop on Petri Nets and Software Engineering (2014), pp.233-248.

Rahim, M., Hammad, A. and Ioualalen, M., Modular and Distributed Verification of SysML Activity Diagrams, Proceedings of the 1st International Conference on Model-Driven Engineering and Software Development (2013), pp.202205.

Ribeiro, Ó. R. and Fernandes, J. M., Some Rules to Transform Sequence Diagrams into Coloured Petri Nets, Proceedings of the 7th Workshop and Tutorial on Practical Use of Coloured Petri Nets and the CPN Tools (2006), pp.237-256.

Saputra, A. B., Basuki, T. A. and Tirtawangsa, J., Transformation of UML 2.0 sequence diagram into Colored Petri Nets, Proceedings of 2014 International Conference of Advanced Informatics: Concept, Theory and Application (2014), pp.243-248.

Sawada, H., Upstream design and 1D-CAE, Journal of System Design and Dynamics, Vol.6, No.3 (2012), pp.351-358.

Tiller, M., Introduction to Physical Modeling with Modelica (2001), Springer.

Tomiyama, T. and Yoshikawa, H., Towards a theory of function-Design perspective, Journal of the Japan Society for Precision Engineering, Vol.56, No.6 (1990), pp.964-968. (In Japanese)

Yang, N., Yu, H., Sun, H. and Qian, Z., Modeling Activity Diagrams with Extended Petri Nets, Intelligent Automation and Soft Computing, Vol.17 (2011), pp.725-735. 